\title{
Comparison of Five Near-Infrared Fluorescent Folate Conjugates in an Ovarian Cancer Model
}

\author{
Elvira García de Jalón ${ }^{1,2}$, Katrin Kleinmanns ${ }^{1}$, Vibeke Fosse ${ }^{1}$, Ben Davidson ${ }^{3}$, Line Bjørge ${ }^{1,4}$, \\ Bengt Erik Haug ${ }^{2}$, and Emmet McCormack ${ }^{1,5,6}$ \\ ${ }^{1}$ Centre for Cancer Biomarkers CCBIO, Department of Clinical Science, The University of Bergen, Jonas Lies vei 65, 5021 Bergen, \\ Norway \\ ${ }^{2}$ Department of Chemistry and Centre for Pharmacy, University of Bergen, Allégaten 41, N-5007 Bergen, Norway \\ ${ }^{3}$ Department of Pathology, Oslo University Hospital, Norwegian Radium Hospital, and Faculty of Medicine, Institute of Clinical Medicine, \\ University of Oslo, Oslo, Norway \\ ${ }^{4}$ Department of Obstetrics and Gynaecology, Haukeland University Hospital, 5021 Bergen, Norway \\ ${ }^{5}$ Centre for Pharmacy, Department of Clinical Science, The University of Bergen, Jonas Lies vei 65, 5021 Bergen, Norway \\ ${ }^{6}$ Vivarium, Department of Clinical Science, The University of Bergen, Jonas Lies vei 65, 5021 Bergen, Norway 2021
}

\begin{abstract}
Purpose: Fluorescence imaging (FLI) using targeted near-infrared (NIR) conjugates aids the detection of tumour lesions pre- and intraoperatively. The optimisation of tumour visualisation and contrast is essential and can be achieved through high tumour-specificity and low background signal. However, the choice of fluorophore is recognised to alter biodistribution and clearance of conjugates and is therefore a determining factor in the specificity of target binding. Although ZW800-1, IRDye ${ }^{\circledR} 800 \mathrm{CW}$ and ICG are the most commonly employed NIR fluorophores in clinical settings, the fluorophore with optimal in vivo characteristics has yet to be determined. Therefore, we aimed to characterise the impact the choice of fluorophore has on the biodistribution, specificity and contrast, by comparing five different NIR fluorophores conjugated to folate, in an ovarian cancer model.

Procedures: ZW800-1, ZW800-1 Forte, IRDye ${ }^{\circledast} 800$ CW, ICG-OSu and an in-house synthesised Cy7 derivative were conjugated to folate through an ethylenediamine linker resulting in conjugates 1-5, respectively. The optical properties of all conjugates were determined by spectroscopy, the specificity was assessed in vitro by flow cytometry and $\mathrm{FLI}$, and the biodistribution was studied in vivo and ex vivo in a subcutaneous Skov-3 ovarian cancer model.

Results: We demonstrated time- and receptor-dependent binding of folate conjugates in vitro and in vivo. Healthy tissue clearance characteristics and tumour-specific signal varied between conjugates 1-5. ZW800-1 Forte (2) revealed the highest contrast in folate receptor alpha (FRa)-positive xenografts and showed statistically significant target specificity. While conjugates 1, $\mathbf{2}$ and $\mathbf{3}$ are renally cleared, hepatobiliary excretion and no or very low accumulation in tumours was observed for $\mathbf{4}$ and $\mathbf{5}$. Conclusions: The choice of fluorophore has a significant impact on the biodistribution and tumour contrast. ZW800-1 Forte (2) exhibited the best properties of those tested, with significant specific fluorescence signal.
\end{abstract}

Key Words Folate · Near-infrared fluorescence imaging · NIRF · Intraoperative navigation · Image-guided surgery · Ovarian · NIR dyes · Pharmacokinetics · Biodistribution · Folate receptor alpha

Correspondence to: Bengt Erik Haug; e-mail: Bengt-Erik.Haug@ uib.no, Emmet McCormack; e-mail: emmet.mc.cormack@uib.no 


\section{Introduction}

Fluorescence imaging (FLI) has developed into a key tool for monitoring tumour progression, invasion and treatment efficacy in preclinical xenograft models [1,2]. In addition, FLI facilitates biological assessment and thus aids the clinical translation of newly developed fluorescent dyes and their corresponding conjugates $[3,4]$ for intra-operative tumour visualisation, often referred to as fluorescence image-guided surgery (FIGS) [5]. Complete tumour resection remains a cornerstone in cancer therapy and is strongly associated with decreased local recurrence rates and prolonged survival [6,7]. Using target-specific fluorescent conjugates, which are composed of a targeting ligand, a linker and a fluorophore, FIGS aims to illuminate diseased tissue, small metastases and vital structures in real time. Thus, FIGS offers enormous potential to assist the surgeon with tumour visualisation, and identification of additional metastases and margins to ultimately achieve complete resection $[8,9]$. A critical aspect for the success of this technique is a high tumour-to-background ratio (TBR), which provides a good contrast between the tumour mass and healthy tissues.

Much effort has been dedicated to identifying tumourspecific targets and fluorescent conjugates that could be explored clinically. Folate receptor alpha $(\mathrm{FR} \alpha)$ is an example of a widely studied tumour-associated molecular target, which is overexpressed in up to $90 \%$ of epithelial ovarian cancers (EOC) [10, 11], among others. Thus, FR $\alpha$ targeting by folate is a good model system for evaluating FLI in EOC. The application of FR $\alpha$-targeting in preclinical molecular imaging demonstrated promising results [12], and has since been clinically assessed for molecular therapy $[13,14]$, in addition to its application in late stage clinical trials for FIGS of several cancers [8, 15]. Coupled with the identification of novel targets, the development of promising targeting ligands has flourished in recent years, including small molecules, peptides, antibodies or antibody fragments $[16,17]$. The ideal fluorescent conjugate needs to exhibit low toxicity, high photochemical stability and fast clearance. To accomplish this, the design of fluorescent conjugates requires special attention, not only in the choice of targeting ligand, but also in the selection of fluorophore and linker. However, as discussed by Debie et al. [16], most of these targeting ligands are often conjugated to readily available fluorophores, such as IRDye ${ }^{\circledR}$ $800 \mathrm{CW}$. Fluorophores with emission in the near-infrared region (NIR; 650-900 $\mathrm{nm}$ ) are preferred due to reduced tissue absorption and increased depth penetration of light. The NIR dye indocyanine green (ICG) has been employed for the assessment of vital structures such as bile ducts location during surgery and for the visualisation of ovarian tumour tissue by passive accumulation $[18,19]$ and/or by enhanced permeability and retention (EPR) effect [20,21]. Due to its clinical approval, ICG has also been employed in targeted approaches [22, 23], although its use has not been extensively accepted due to the challenges with conjugation of this fluorescent dye to targeting ligands. Moreover, the fluorescence of ICG is known to quench after conjugation to proteins, which may have a detrimental impact on its use for imaging purposes [24]. Besides IRDye ${ }^{\circledR} 800 \mathrm{CW}$ and ICG, other commercially available NIR dyes are in different stages of development for FIGS [16, 17]. Various fluorophores are based on the cyanine scaffold, and are decorated with a variety of functional groups, which results in different pharmacological properties [25]. Such differences in physicochemical properties of the NIR fluorophores also have a major impact on the target binding specificity of their conjugates since, e.g. biodistribution and clearance can be affected with several cases previously reported where the choice of fluorophore, or linker, affected the affinity of the targeting ligand to the molecular target of interest [26-28]. Those studies highlight the importance of reducing background signal, improving contrast during FLI by avoiding non-specific binding, improving stability and enhancing clearance from the body.

Using folate as a ligand to target FR, we aimed to investigate the impact of NIR fluorophores on biodistribution, specificity and sensitivity in a comparison study of five fluorescent conjugates. We conjugated four commercially available fluorescent dyes with similar spectral properties and clinical translational potential, namely ZW800-1 (1), ZW800-1 Forte (2), IRDye ${ }^{\circledR} 800 \mathrm{CW}$ (3) and ICG-OSu (4), and one in-house synthesised $\mathrm{Cy} 7$ dye (5), to folate through an ethylenediamine (EDA) linker (see Fig. 1 for structures) and evaluated the different conjugates in subcutaneous EOC xenograft models.

\section{Materials and Methods}

\section{Synthetic Procedures}

$\gamma$-Ethylenediamine folate (EDAF) was reacted with the NHS esters of ZW800-1 (Curadel LCC, MA, USA), ZW 800-1 Forte (Curadel LCC), IRDye ${ }^{\circledR} 800 \mathrm{CW}$ (LICOR, NE, USA), ICG-OSu (AAT Bioquest, CA, USA) and an in-house synthesised Cy7 derivative, to give conjugates 1-5, respectively. Detailed synthetic and analytical data are given in the electronic supplementary material.

\section{Fluorescence Spectroscopy}

The spectral characteristics of the conjugates $\mathbf{1 - 5}$ were determined using a Spark 20M plate reader (Tecan, Switzerland). The emission and excitation of all conjugates were measured in triplicates with a bandwidth of $5 \mathrm{~nm}$ in a 96-well Half-Area Microplate at a concentration of $100 \mu \mathrm{M}$ dissolved in phosphate buffered saline (PBS). The 
fluorescence intensity was plotted as normalised relative fluorescence units (RFU).

\section{Cell Lines and Cell Culture}

Human EOC Skov-3, lung carcinoma A549 and cervical adenocarcinoma HeLa cell lines were employed to study FR $\alpha$ expression. Cells were cultured in DMEM (Sigma-Aldrich, MO, USA) supplemented with $10 \%$ FBS and $1 \%$ L-glutamine (Sigma-Aldrich) and kept at $37{ }^{\circ} \mathrm{C}$ and $5 \% \mathrm{CO}_{2}$. For the binding and specificity assay, cells were cultured in folic acid free medium.

\section{In Vitro Validation: Flow Cytometry, Optical FLI and Cytotoxicity}

The degree of FR $\alpha$ binding in Skov-3, A549 and HeLa cells was quantitatively determined and expressed as antibodybinding capacity $(\mathrm{ABC})$ units, employing a bead calibration kit (Qifikit, Dako Agilent, CA, USA). $1 \times 10^{5}$ cells were re-suspended in $100 \mu \mathrm{L}$ PBS $+2 \%$ bovine serum albumin (BSA) buffer and stained with a saturated concentration of $5 \mu \mathrm{g}$ unconjugated mouse anti-human FR $\alpha$ (Clone 548908, Thermo Fisher Scientific, MA, USA) for $45 \mathrm{~min}$ at $4{ }^{\circ} \mathrm{C}$. An unconjugated monoclonal CD33 IgG1 antibody (Clone WM53, 1:20 Bio-Rad, UK) was used as a negative control. Cells, set-up beads and calibration beads were each washed twice with PBS-BSA-Azide buffer and stained with a secondary FITC-conjugated antibody (provided in the Qifikit, 1:50) for $45 \mathrm{~min}$ at $4{ }^{\circ} \mathrm{C}$. After two further washing steps, samples were acquired and recorded with the BD Fortessa flow cytometer (BD Biosciences, NJ, USA), with a laser excitation at $488 \mathrm{~nm}$ and BP emission filter at $530 \pm 15 \mathrm{~nm}$.

The binding and specificity to FR $\alpha$ of conjugates 1-5 was determined by FLI in (1) FR $\alpha$ low, intermediate and high expressing cell lines and (2) different conjugate concentrations with or without the addition of folic acid (FA). First, $5 \times 10^{6}$ A549 (negative control), HeLa (positive control) and Skov-3 cells were incubated with $10 \mu \mathrm{M}$ of conjugate 1-5. Secondly, $7.5 \times 10^{5} \mathrm{HeLa}$ cells were incubated with 10,1 or $0.1 \mu \mathrm{M}$ of conjugates $\mathbf{1 - 5}$ in the presence or absence of 100-fold molar excess of FA. For both experiments, cells were incubated for $4 \mathrm{~h}$ at $37^{\circ} \mathrm{C}$, washed twice, centrifuged and kept in cell pellets without supernatant. Fluorescence images were acquired with the IVIS Spectrum In Vivo Imaging System (PerkinElmer Inc., MA, USA) using the filter settings $\lambda_{\mathrm{ex}}=745 \pm 15 \mathrm{~nm}, \lambda_{\mathrm{em}}=800$ $\pm 10 \mathrm{~nm}$ and signals were expressed as average radiance $\left(\mathrm{p} / \mathrm{s} / \mathrm{cm}^{2} / \mathrm{sr}\right)$.

The toxicity of all five conjugates was tested in vitro. Skov-3 cells were cultured in triplicate and incubated for $4 \mathrm{~h}$ with $0.1-100 \mu \mathrm{M}$ of each conjugate, before cell proliferation was assessed using a water-soluble tetrazolium salt assay (WST-1, Roche applied Science, Germany). The metabolite formazan was measured spectrophotometrically to determine the metabolically active cells in each well. The formazan concentration of each sample was normalised to Skov-3 cells cultured without the addition of a conjugate.

\section{Subcutaneous Xenograft Models}

All applicable institutional and/or national guidelines for the care and use of animals were followed. All experiments were approved by The Norwegian Animal Research Authority and conducted according to The European Convention for the Protection of Vertebrates Used for Scientific Purposes (Application ID 14128). NOD-scid IL2Rg ${ }^{\text {null }}$ mice (referred to as NSG) were housed in groups of $\leq 5$ in individually ventilated cages changed twice per month and kept in a 12-h dark/night schedule at $2{ }^{\circ} \mathrm{C}$ at the Vivarium, University of Bergen. Observations for general condition and body weights were recorded twice per week and, when indicated, the animals were euthanised according to institutional guidelines. Skov-3 and A549 subcutaneous xenografts were established through bi-lateral flank injections of $5 \times 10^{6}$ cells, resuspended in $100 \mu \mathrm{L}$ PBS containing 16.7\% Matrigel (Corning Inc., MA, USA). Tumour volumes were measured weekly with a digital calliper and calculated using the ellipsoid volume formula: Volume $=\pi$ (length $\times$ width $\times$ height $) / 6$.

\section{Optical Imaging of the Subcutaneous Xenografts}

\section{In Vivo Biodistribution and Optimal Time Point Imaging}

For biodistribution assessment of conjugates 1-5, mice were intravenously (iv) injected with $100 \mu \mathrm{L}$ of a $10-\mu \mathrm{M}$ conjugate solution when mean tumour volumes reached $\approx 150 \mathrm{~mm}^{3}$, corresponding to a dose of $500 \mathrm{nmol} / \mathrm{mouse}$. The dose was chosen on the basis of preclinical mouse models previously performed using folate-NIR (OTL38) FLI [28]. To determine the timepoint for the optimal TBR, optical imaging was performed at $0 \mathrm{~h}, 0.5 \mathrm{~h}, 1 \mathrm{~h}, 2 \mathrm{~h}, 4 \mathrm{~h}, 6 \mathrm{~h}, 8 \mathrm{~h}, 24 \mathrm{~h}$ and $48 \mathrm{~h}$ using the IVIS Spectrum. Fluorescence signals were analysed with the Living Imaging ${ }^{\circledR}$ software v4.5 (PerkinElmer Inc.) and expressed as average radiance $\left(\mathrm{p} / \mathrm{s} / \mathrm{cm}^{2} / \mathrm{sr}\right)$. Regions of interest (ROI) were manually drawn around the subcutaneous tumours in the flanks and the adjacent tissue to calculate TBRs at all time points. Next, subcutaneous xenograft models were imaged at the pre-determined optimal time points, which were $4 \mathrm{~h}$ for conjugates $\mathbf{1}, \mathbf{2}$ and $\mathbf{3}$ and $24 \mathrm{~h}$ for conjugates $\mathbf{4}$ and $\mathbf{5}$.

\section{Ex Vivo Biodistribution}

Organ biodistribution was assessed ex vivo for each conjugate in Skov-3 and in the control cell line, A549. Four hours 
b

1

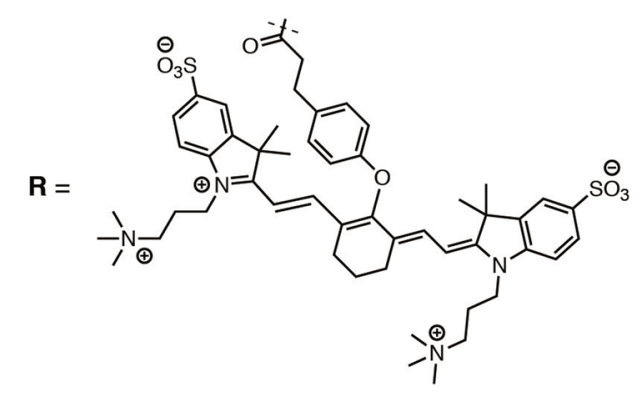

2<smiles>CC1(C)C(=CC=CC2=C(c3ccc(CCC(=O)[O-])cc3)C(=CC=C3N(CCC[N+](C)(C)C)c4ccc([O-])cc4C3(C)C)CCC2)N(CCC[N+](C)(C)C)c2ccc([O-])cc21</smiles>

3

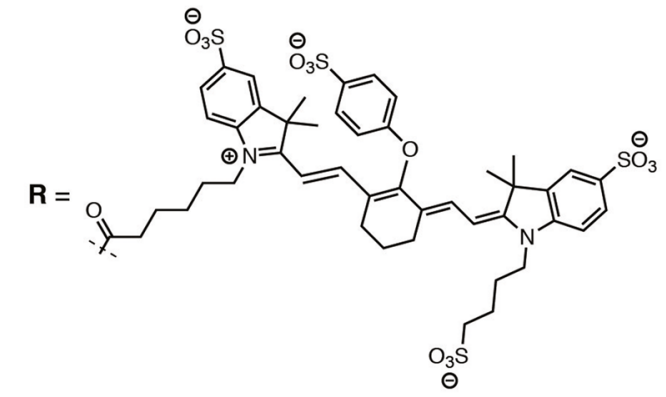

4

$\mathbf{R}=$<smiles>CC(C)=O</smiles>

$5 \quad \mathbf{R}=$<smiles>CC(=O)CCCCN1C(=CC=C2CCCC(C=CC3(C)CN(C)c4ccccc43)C2Cl)C(C)(C)c2ccccc21</smiles><smiles>[R]NCCNC(=O)CCC(NC(=O)c1ccc(NCc2cnc3nc(N)[nH]c(=O)c3n2)cc1)C(=O)O</smiles>

C
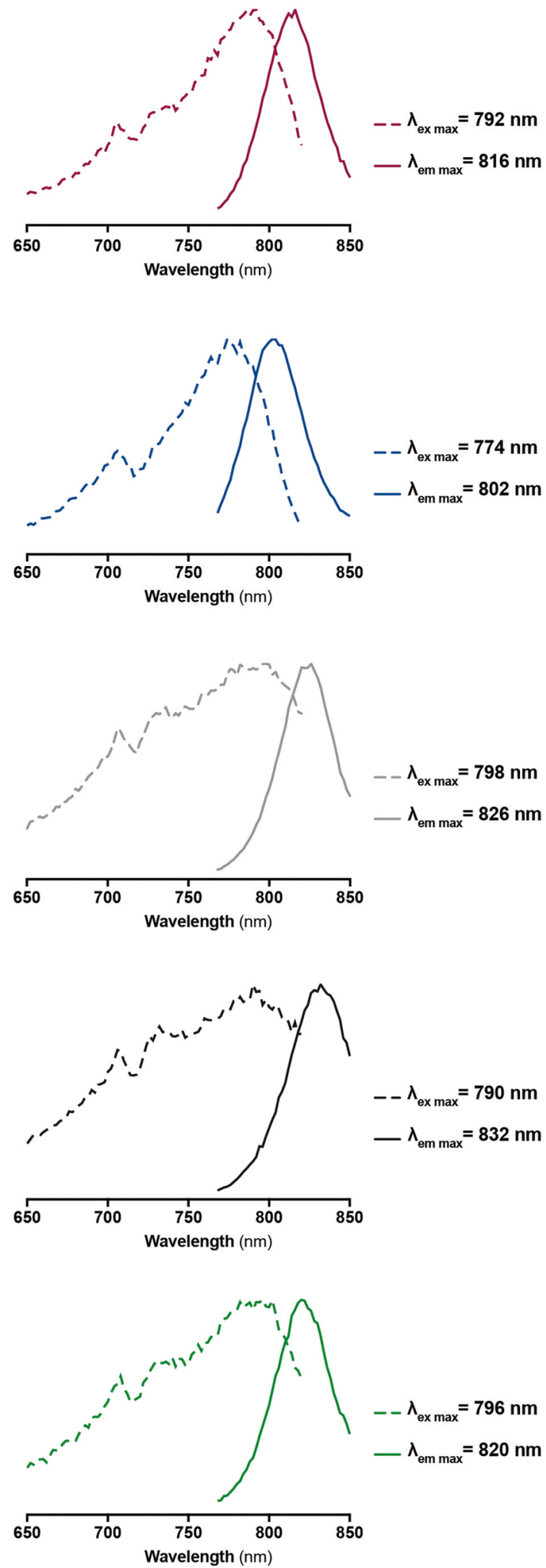
4 Fig. 1. Chemical structure and optical properties of five farred shifted folate conjugates. All conjugates were synthesised to target the FRa through the folate ligand. (a) The conjugation of folate (depicted in black) through an ethylenediamine linker (EDA, depicted in red) to (b) five fluorescent dyes was performed. (c) Spectral properties including peak emission and excitation of conjugates 1-5 dissolved in DMSO.

after iv administration of $\mathbf{1}, \mathbf{2}$ and $\mathbf{3}$ and $24 \mathrm{~h}$ after injection of 4 and $\mathbf{5}(100 \mu \mathrm{l}, 10 \mu \mathrm{M})$, mice were euthanised and tumours and organs were harvested. FLI was performed with the IVIS Spectrum, and subsequently, fluorescence was acquired using the FLARE® intraoperative system (Curadel LLC), employing the NIR channel \#2 $\left(\lambda_{\mathrm{ex}}=760 \pm 3 \mathrm{~nm}\right.$ and $\lambda_{\mathrm{em}}=>781$ $\mathrm{nm}$ longpass) at three different exposure times (500, 1000 and $2000 \mathrm{~ms}$ ) at a gain of 1. IVIS Spectrum data were analysed as previously described, and FLARE data were analysed using ImageJ (Fiji, version 1.52p, [29]) and the integrated FLARE analysis platform using grey scale values expressed in arbitrary units (AU).

\section{Immunohistochemistry}

Resected tumours of A549 and Skov-3 were fixed in 4\% paraformaldehyde, embedded in paraffin(FFPE) and immunohistochemically stained as described earlier [30]. In short, 5 - $\mu \mathrm{m}$ sectioned FFPE samples were stained with anti-human $\mathrm{FR} \alpha$ (clone SA170417DD, 1:2000, Invitrogen) and evaluated using an immunoreactivity scoring system defined by the product $\left(I^{*} E\right)$ with $I$ being the staining intensity $(0=$ negative, $1=$ weak, $2=$ moderate) and $E$ being the staining extent $(0=0 \%, 1=1-5 \%, 2=6-25 \%, 3=26-75 \%, 4=$ $76-100 \%$ ) resulting in a score of $0-8$. Rabbit-specific polymer-horseraddish peroxidase was used to avoid false-positive cross-specific bindings in mouse tissue.

\section{Statistics}

Results are given as mean \pm standard deviation (SD). After randomisation, a one-way ANOVA was applied to ensure unbiased assignment of tumour volumes among the experimental groups. Comparison of means was performed using Student $t$-tests. All statistical tests were performed using GraphPad Prism v 6.0h (GraphPad Software Inc, CA, USA) and $p<0.05$ was considered significant.

\section{Results}

\section{Synthesis, Purification and Optical Properties Determination of Cy7 Conjugates}

Fluorophore choice is known to influence the tumour-specific fluorescence signal due to unspecific accumulation and distribution in the body [26]. To investigate which fluorescent dye favours tumour-specific optical imaging in EOC xenograft models, we conjugated five different fluorophores (the commercially available ZW800-1, ZW800-1 Forte, IRDye ${ }^{\circledR}$ 800CW and ICG-OSu, and one in-house synthesised Cy7) to the well-established targeting ligand folate, utilising an ethylenediamine (EDA) linker. The chemical structure of the resulting five different fluorescent conjugates, $\mathbf{1 - 5}$, is depicted in Fig. 1b. Detailed descriptions of the different synthetic approaches performed to obtain the targeting ligand and conjugates $\mathbf{1 - 5}$, as well as the corresponding analytical data (Fig. S1 to S15), $\log \mathrm{D}$ and surface charge distribution (Fig. S16), can be found in the electronic supplementary material.

Using conjugates $\mathbf{1 - 5}$, we evaluated how the conjugation of the different fluorophores to EDAF (Fig. 1a) affected the optical properties. To do so, we defined their individual excitation and emission spectra, and determined the optimal FLI parameters. The different conjugates (Fig. 1b) were diluted in medium or in DMSO and the peak excitation and emission obtained are reported in Table 1, Fig. 1c and Fig. S17.

\section{Folate Receptor Alpha Expression and Conjugate Binding}

Next, we investigated the expression of FR $\alpha$ in the EOC cell line Skov-3 prior to in vivo FLI. We quantified the FR $\alpha$ binding sites of Skov-3 using an antibody-based flow cytometry approach and compared it to the expression profiles of the highly FR $\alpha$-positive endometrial cancer cell line HeLa and

Table 1 Summary of the spectroscopic properties of conjugates 1-5 measured in medium and DMSO

\begin{tabular}{|c|c|c|c|c|c|c|}
\hline \multirow[t]{2}{*}{ Conjugate } & \multicolumn{3}{|c|}{ Culture medium } & \multicolumn{3}{|l|}{ DMSO } \\
\hline & $\lambda_{\mathrm{ex}}(\mathrm{nm})$ & $\lambda_{\mathrm{em}}(\mathrm{nm})$ & Stokes shift (nm) & $\lambda_{\mathrm{ex}}(\mathrm{nm})$ & $\lambda_{\mathrm{em}}(\mathrm{nm})$ & Stokes shift (nm) \\
\hline 1 & 777 & 802 & 25 & 792 & 816 & 24 \\
\hline 2 & 760 & 788 & 28 & 774 & 802 & 28 \\
\hline 3 & 783 & 808 & 25 & 798 & 826 & 28 \\
\hline 4 & 784 & 820 & 36 & 790 & 832 & 42 \\
\hline 5 & 789 & 804 & 15 & 796 & 820 & 24 \\
\hline
\end{tabular}




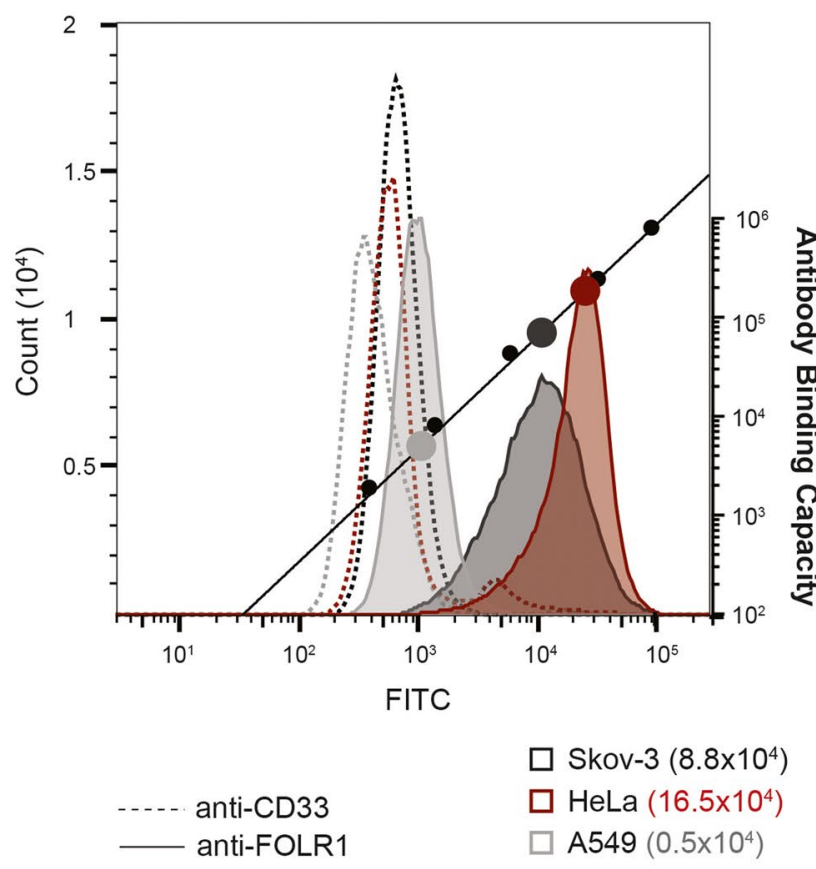

b

1

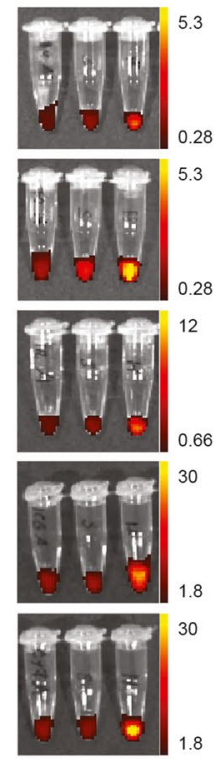

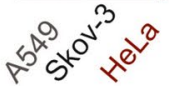

C

Conjugates 1 - 5

\begin{tabular}{lll}
\hline $10 \mu \mathrm{M}$ & $1 \mu \mathrm{M}$ & $0.1 \mu \mathrm{M}$
\end{tabular}

1

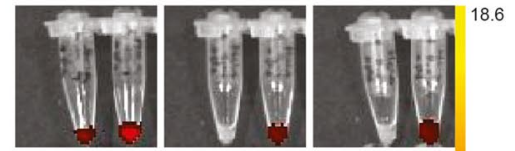

2

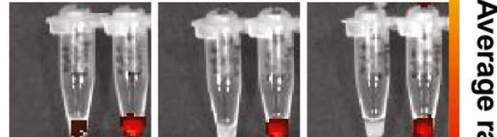

3

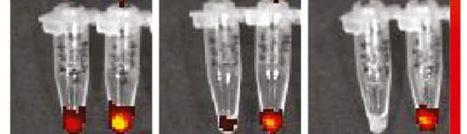

4

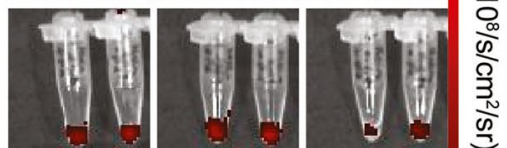

5

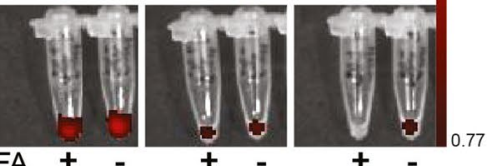

HeLa

d

$10 \mu \mathrm{M}$

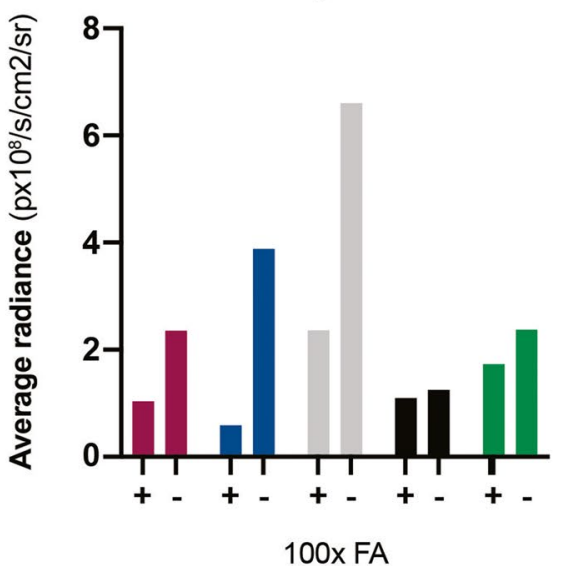

$1 \mu \mathrm{M}$

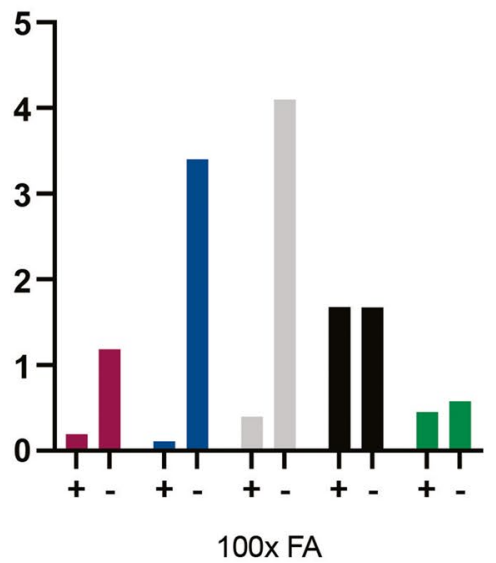

$0.1 \mu \mathrm{M}$

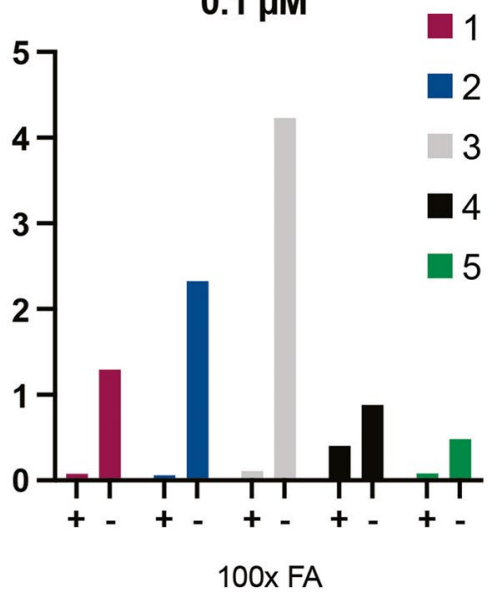

Fig. 2. Folate receptor alpha ( $F R a)$ expression and conjugate binding affinity in three human tumour cell lines. a The antibody binding capacity of the ovarian cancer cell line Skov-3 was evaluated and compared to the FRa-positive control cell line HeLa and negative control cell line A549. Flow cytometry fluorescence intensity of anti-FOLR1-FITC cancer cell lines was correlated to the antibody binding capacity $(A B C)$ of calibration beads. The resulting $A B C$ was calculated and presented for each cell line. An anti-CD33 monoclonal antibody served as a negative control. $\mathbf{b}$ For the validation of target-specific binding of all five conjugates, stained cell line pellets $(5 \times$ $10^{6}$ cells) were imaged at the IVIS spectrum. Data reported as average radiance $\left(\mathrm{p} \times 10^{8} / \mathrm{s} / \mathrm{cm}^{2} / \mathrm{sr}\right)$. c HeLa cell line pellets stained with 10,1 and $0.1 \mu \mathrm{M}$ of conjugates 1-5 in the presence or absence of 100-fold molar excess folic acid (FA) imaged at the IVIS spectrum. $\mathbf{d}$ Quantification (ROI) of fluorescence signals from (c) reported as average radiance $\left(\mathrm{p} \times 10^{8} / \mathrm{s} / \mathrm{cm}^{2} / \mathrm{sr}\right)$.

the FR $\alpha$ low expressing lung cancer cell line A549 (Fig. 2a). Skov-3 revealed an average expression of $8.8 \times 10^{4} \mathrm{FR} \alpha$ ABC/cell, compared to the $0.5 \times 10^{4}$ obtained for A549, confirming FR $\alpha$ abundance (Fig. 2a). For the evaluation of the specific binding capacity and possible steric interferences in the binding to the FR $\alpha$ after conjugation, we incubated 5 $\times 10^{6}$ cells per cell line with $10 \mu \mathrm{M}$ of each conjugate (1-5) for $4 \mathrm{~h}$. Fluorescence signals were obtained from all different cell line pellets at the IVIS Spectrum, with the highest fluorescence intensities exhibited by the HeLa cells for all five conjugates (Fig. 2b), consistent with the high FR $\alpha$ expression (16.5 $\times 10^{4} \mathrm{ABC} /$ cell) (Fig. 2a). In addition, the specific 
binding of conjugates 1-5 was assayed in the presence and absence of 100-fold molar excess of folic acid (FA) at 10,1 or $0.1 \mu \mathrm{M}$, using HeLa cells. In the presence of FA, the intensity of the fluorescence was greatly reduced, especially for the lowest concentrations (Fig. 2c, d and S18). In addition, no cytotoxicity or inhibition of cell proliferation was observed at the employed dose for any of the conjugates (Fig. S19).

\section{Biodistribution of Folate Conjugates in Skov-3 Subcutaneous Xenograft Model}

To assess the impact of the different fluorophores in conjugates 1-5 on the tumour-specificity and sensitivity, subcutaneously engrafted NSG mice ( $n=3$ per conjugate) were imaged at eight different time points from 0.5 to 48 h (Fig. 3a). Tumour-specific fluorescence was observed for all conjugates except 4 (Fig. 3a). Conjugate 2 exhibited an intense fluorescence signal in Skov-3 tumours early after iv injection that was sustained up to $48 \mathrm{~h}$. High, non-specific accumulation in healthy tissue at early time points was observed for all conjugates, followed by different time- and excretion route-dependent elimination from the body. Mice injected with conjugates $\mathbf{1}$ and $\mathbf{3}$ exhibited residual fluorescence signal in the kidneys at $24 \mathrm{~h}$ (Fig. 3a white-dashed circles). For conjugates $\mathbf{4}$ and $\mathbf{5}$, very low fluorescence intensity signals were obtained at any given time, with evidence of hepatic excretion (Fig. S20) observed as residual liver fluorescence for $\mathbf{4}$. Interestingly, for conjugate $\mathbf{5}$, the tumour fluorescence was not evident until $4 \mathrm{~h}$ post-injection, but then increased over time to reach the maximum TBR at $24 \mathrm{~h}$ postinjection. It should be noted that conjugate $\mathbf{1}$ employed for the in vivo experiments is estimated to have contained free dye in a ratio of 3:1 (conjugate 1, free dye) (see Fig. S21). Following evaluation of conjugate biodistribution over time, the optimal time point for achieving the best TBR, expressed as the ratio between tumour-specific accumulation and surrounding fluorescence, was determined for each conjugate and was as follows: $\mathbf{1}, 8 \mathrm{~h}$ (TBR $2.0 \pm 0.4$ ); $\mathbf{2}, 8 \mathrm{~h}$ (TBR $2.0 \pm 0.2$ ); $\mathbf{3}, 8$ $\mathrm{h}$ (TBR $1.4 \pm 0.3$ ); 4, $0.5 \mathrm{~h}$ (TBR $1.3 \pm 0.3) ; 5,24$ h (TBR 2.1 \pm 0.6 ) (Fig. 3b). Optimal time points at $8 \mathrm{~h}$ are unpractical for imaging purposes, limiting its applicability. For practicality, 4 $\mathrm{h}$ were selected for imaging, as acceptable TBR values were obtained at this time point, for conjugates 1 (TBR $1.9 \pm 0.3$ ), 2 (TBR $1.8 \pm 0.2$ ) and $\mathbf{3}$ (TBR $1.2 \pm 0.2$ ). For conjugates 4 (TBR $1.0 \pm 0.1$ ) and 5, $24 \mathrm{~h}$ was chosen (Fig. 3b).

\section{Targeting Ligand Specificity and Ex Vivo Biodistribution}

To validate tumour-specific signal intensities, we assessed in vivo and ex vivo FLI in subcutaneous xenografts of Skov-3 and A549 at the optimal time points. Four hours after iv injection of conjugates $\mathbf{1}, \mathbf{2}$ and 3 , and $24 \mathrm{~h}$ after administration of conjugates $\mathbf{4}$ and $\mathbf{5}$, in vivo images were acquired for both groups using the IVIS Spectrum. As seen in Fig. 4a, the signal intensity obtained for all conjugates was higher in Skov-3 tumours compared to xenografted A549 tumours, except for $\mathbf{3}$, presumably due to high background signal. A significant difference in average radiance between Skov-3 and A549 was obtained for conjugate $2(p<0.05)$ (Fig. 4b). FR $\alpha$ immunoreactivity score $(0-8)$ was assessed by IHC in xenografted Skov-3 $(4.6 \pm 2.5)$ and A549 $(2.7 \pm 0.8)$ tumours (Fig. 4c), confirming an association between higher fluorescence intensities in Skov-3 tumours and higher target expression, which was also previously observed by flow cytometry and in vitro FLI (Fig. 2 and S18). However, the standard deviation demonstrates heterogenous expression of FR $\alpha$ in both Skov-3 and A549 xenografts, as already demonstrated for other models [28] (Fig. 2a and 4c).

Ex vivo fluorescence analysis of tumour tissues reveals signal in all Skov-3 tumours except $\mathbf{4}$, with a lower overall intensity in A549 (Fig. 4d). The biodistribution to the kidneys of conjugates $\mathbf{1}, \mathbf{2}$ and $\mathbf{3}$ suggests higher hydrophilicity, translated into renal clearance (Fig. $4 \mathrm{~d}$ and e). Consistently low fluorescent background signals are shown for $\mathbf{4}$ and $\mathbf{5}$, with accumulation of $\mathbf{4}$ mainly in the liver and residual fluorescence signal of 5 in the stomach (Fig. $4 \mathrm{~d}$ and e).

Finally, the fluorescence of all conjugates was evaluated using a clinically compatible intraoperative NIR-FLI system, to assess their suitability as contrast agents for FIGS. The fluorescence signal from Skov-3 tumours, kidneys and liver confirmed the results obtained with the IVIS Spectrum (Fig. 5). As shown in Fig. 5a, the fluorescence signal in tumours, acquired with an exposure time of $2000 \mathrm{~ms}$, was minimal for conjugates 4 and $5(56.4 \pm 4.1$ and $60.9 \pm 6.3 \mathrm{AU}$, respectively), rendering them ineffectual for in vivo evaluation of FR $\alpha$ expression. In addition, we observed low fluorescence in the kidneys $(100.4 \pm 37.8$ and $63.9 \pm 11.3 \mathrm{AU}$, respectively, Fig. 5b) for both conjugates, but high fluorescence intensity in the liver of mice injected with $4(283.3 \pm 38.7$ AU, Fig. 5c). Tumour-specific fluorescence was observed for conjugate $1(156.9 \pm 22.0 \mathrm{AU}), 2(213.1 \pm 51.0 \mathrm{AU})$ and $3(189.7 \pm 27.1 \mathrm{AU})$ (Fig. 5a). The ex vivo comparison of signal intensities in the intraoperative setting of Skov-3 and A549 xenografted tumours revealed increased fluorescence in Skov-3 tumours for all conjugates (Fig. S22), confirming the ex vivo results obtained from the IVIS Spectrum. While fluorescence values in the liver were below the ones obtained for the tumours, fluorescence in the kidneys was higher than in the tumours in all cases, with the highest kidney intensities obtained for 3, 913.6 \pm 248.9 AU (Fig. 5b and c).

\section{Discussion}

Substantial differences in the optical and targeting properties of conjugates formed by the same targeting ligand, but with different linkers or fluorophores, have been described $[26,28]$. In addition, the choice of fluorophore has a major impact on tumour contrast due to its influence on biodistribution and clearance [31]. This means that the physicochemical properties of the fluorophore also need to be taken into 
a

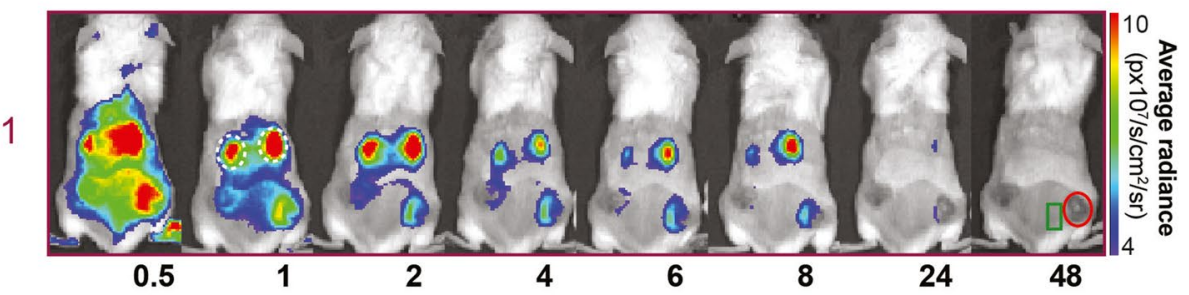

2

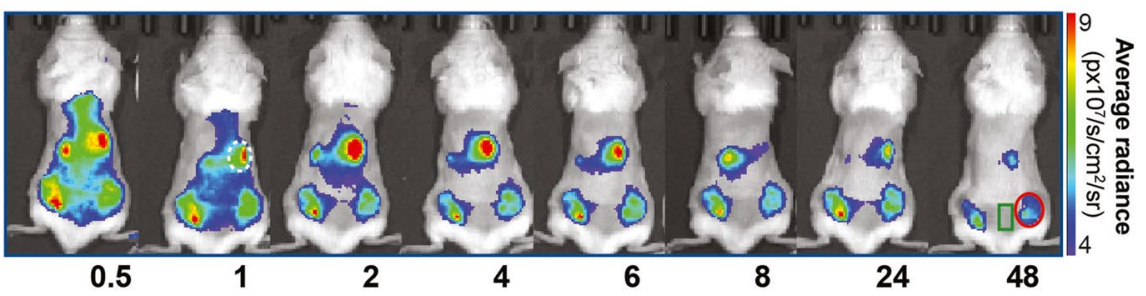

3

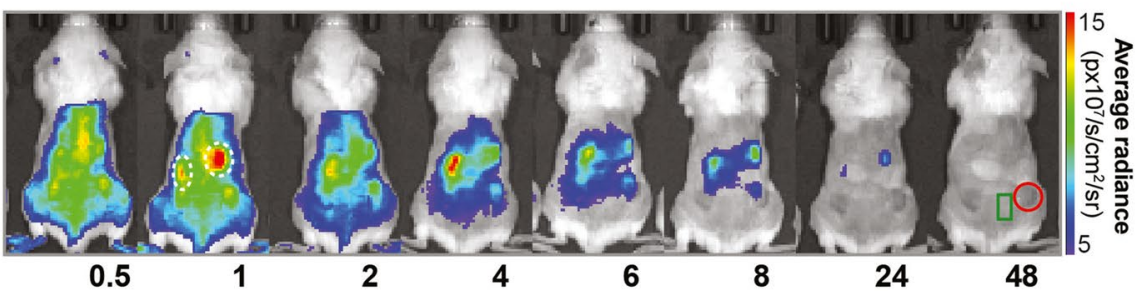

4

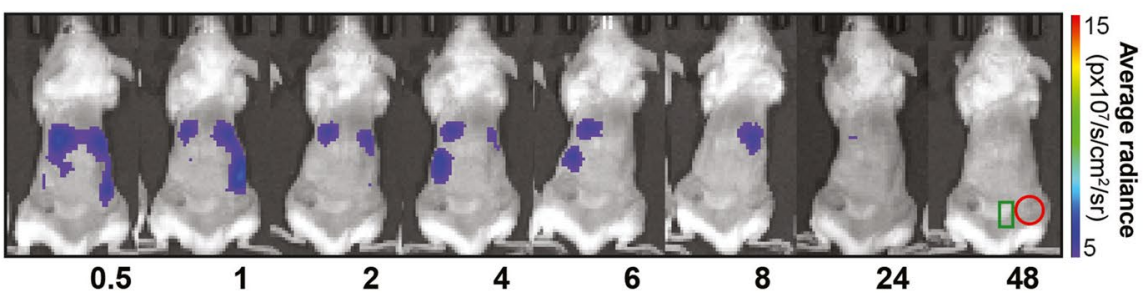

5

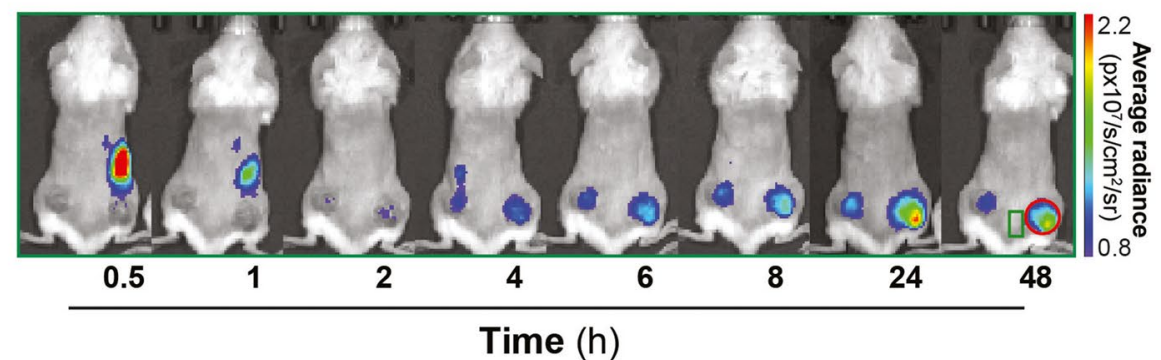

b
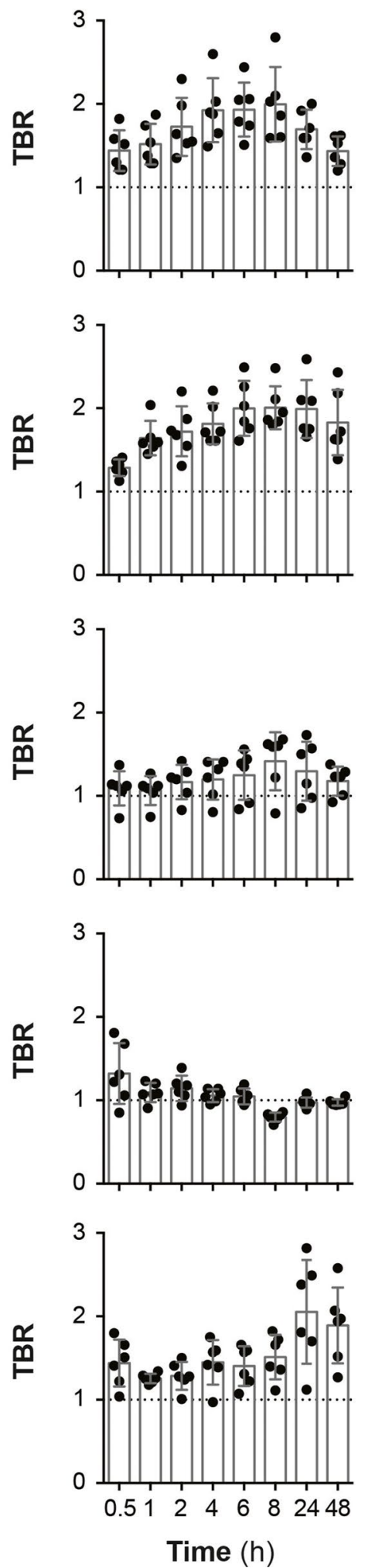

Fig. 3. Assessment of conjugate biodistribution in subcutaneous Skov-3 xenograft models. (a) Longitudinal in vivo whole body optical FLI at eight different time points $(0.5 \mathrm{~h}, 1 \mathrm{~h}, 2 \mathrm{~h}, 4 \mathrm{~h}, 6 \mathrm{~h}, 8 \mathrm{~h}, 24 \mathrm{~h}$ and $48 \mathrm{~h})$ of one representative mouse $(n=3)$. The fluorescence signal in the tumours (red circle), surrounding tissue (green rectangle) and kidneys (dashed white circle, when required) of each conjugate 1-5 (which were based on ZW800-1, ZW800-1 Forte, IRDye ${ }^{\circledast} 800 C W$, ICG-OSu and one in-house synthesised dye, respectively) was quantified and (b) presented as tumour to background ratios (TBR) for all time points. 
a

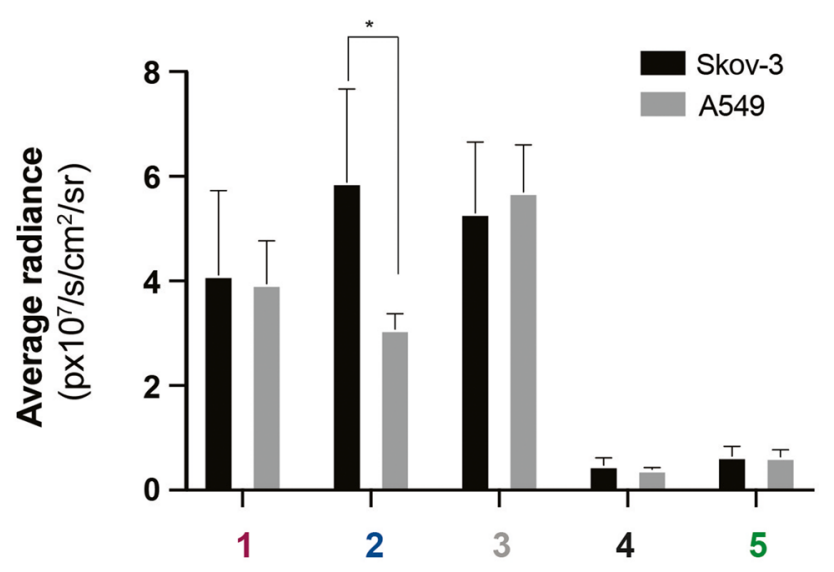

d
1

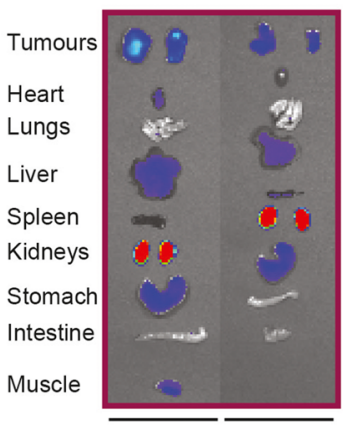

Skov-3 A549 b

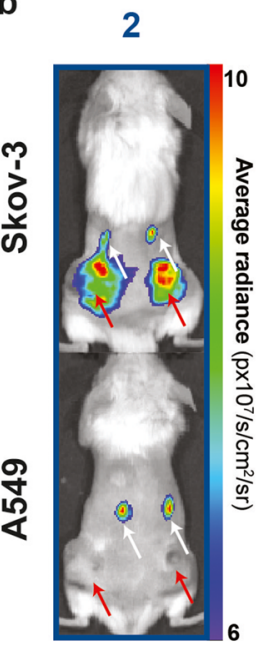

c
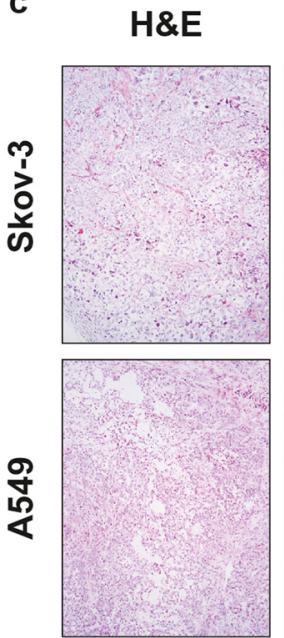

FOLR1 IHC
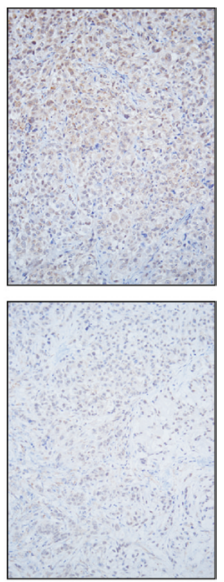

5

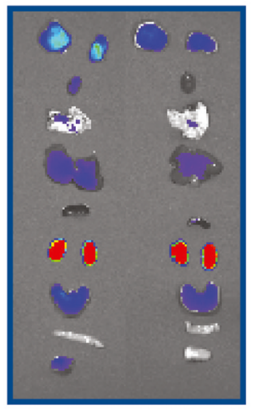

3

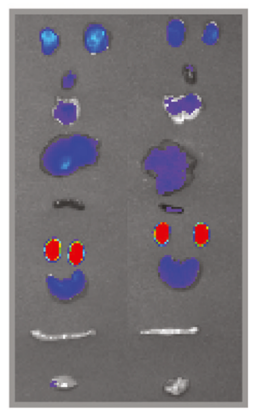

4
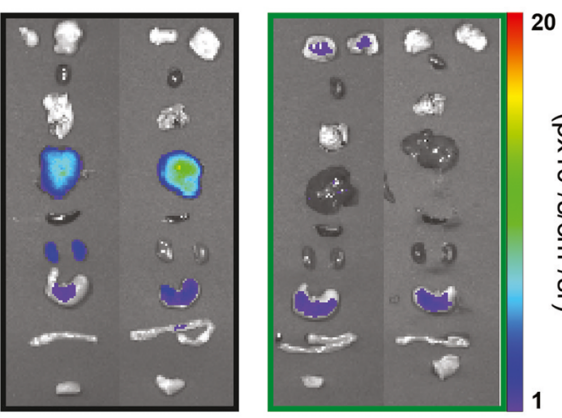

20

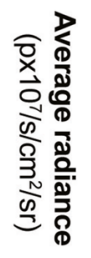

e

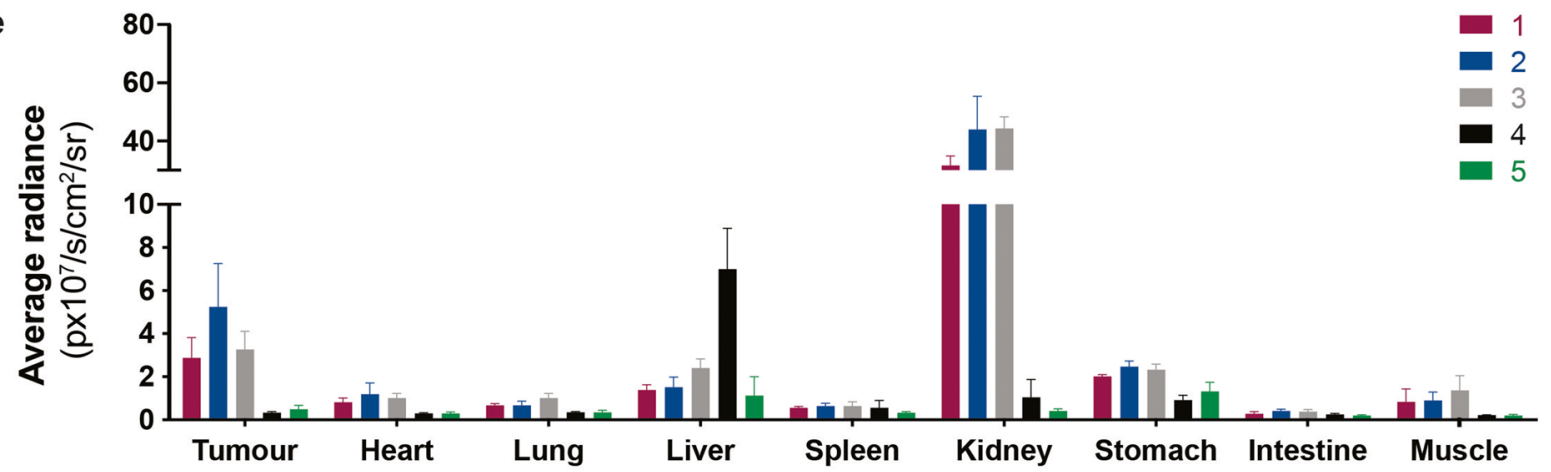

Fig. 4. Quantification of average fluorescence intensities in Skov-3 and A549 subcutaneous xenografts. (a) By quantifying the in vivo fluorescence intensities of all five conjugates in Skov-3 (n= 8-14) and A549 (n=2-4) at 4h for 1-3 and 24h for 4 and 5, FRa expressiondependent accumulation in the tumour was observed. (b) Significant increased tumour fluorescence signal in Skov-3 compared to A549 4h after ZW800-1 Forte (2) injection. The fluorescence signal in tumours and in kidneys is highlighted with red and white arrows, respectively. (c) The expression and distribution of FRa were assessed by IHC in both xenografted cell line tumour samples (Skov-3 $n=12$ and A549 $n=6$ ). Sectioned tumour samples were additionally stained with haematoxylin and eosin. (d) Ex vivo tumour and organ average fluorescence intensities of Skov-3 and A549 were quantified (e) to assess the conjugate accumulation at the optimal time point determined for 1-5 (based on ZW800-1, ZW800-1 Forte, IRDye ${ }^{\circledR} 800 \mathrm{CW}$, ICG-OSu and one in-house synthesised Cy7 dye, respectively). Statistical analysis (one-way ANOVA with unpaired Mann-Whitney $U$ test) with $p<0,05\left(^{*}\right)$ was regarded as statistically significant. 
a

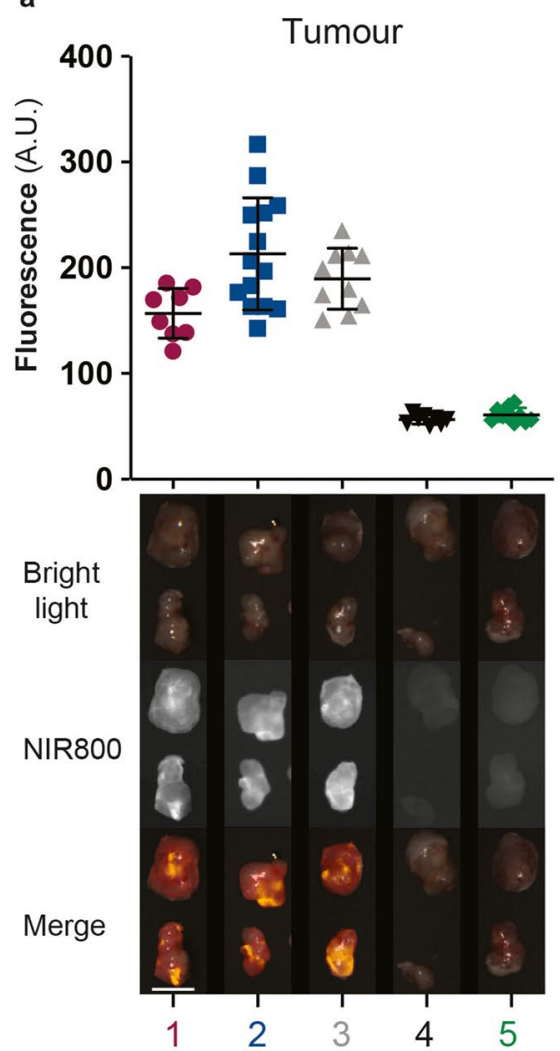

b

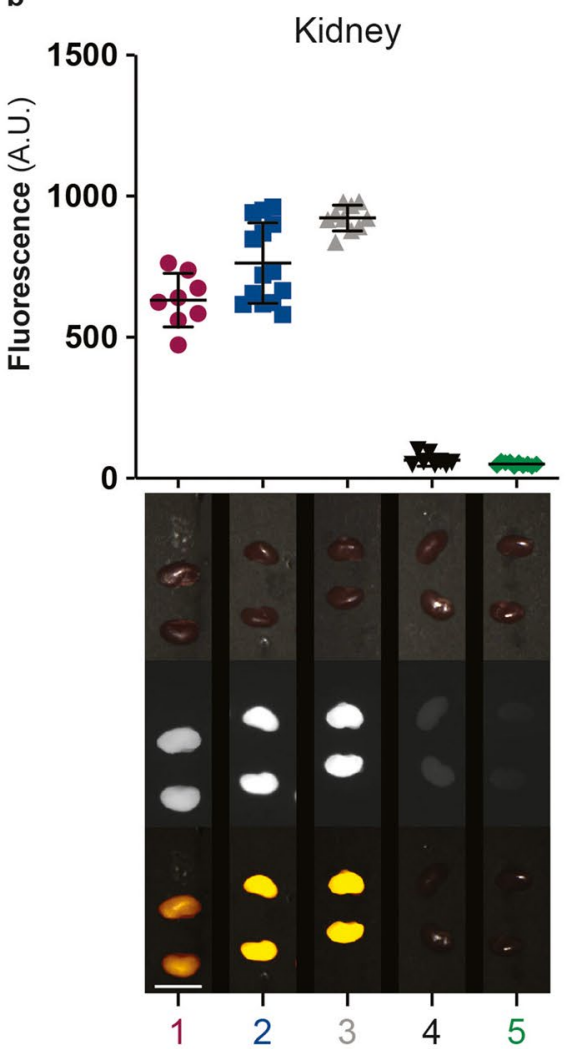

C

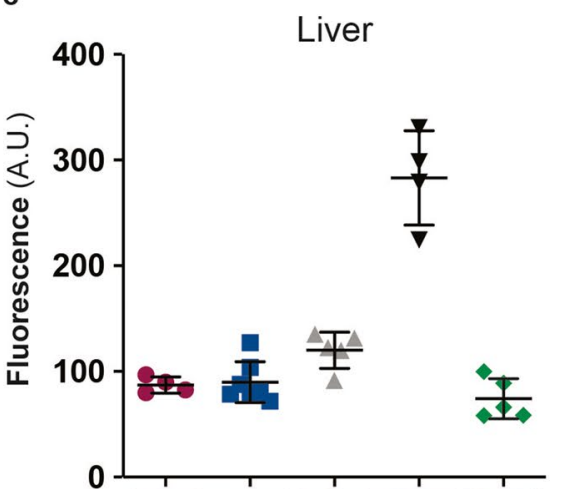

Fig. 5. Intraoperative assessment of conjugates in Skov-3 xenografts. Ex vivo fluorescence intensities for (a) tumour (2000 ms exposure time), (b) kidney (1000 ms exposure time), and (c) liver (2000 ms exposure time) presented in arbitrary greyscale units (A.U.) (1 $n=$ $8 ; \mathbf{2} n=14 ; \mathbf{3} n=10 ; \mathbf{4} n=8 ; \mathbf{5} n=10$, at $4 \mathrm{~h}$ for $\mathbf{1 - 3}$ and $24 \mathrm{~h}$ for $\mathbf{4}$ and 5). Representative colour, near-infrared (NIR) 800 and pseudocoloured fluorescent signal merge image of the subcutaneous tumour, kidney and liver. (Scale bars $1 \mathrm{~cm}$ ).

consideration, in addition to the fluorescent optical properties such as large Stokes shifts, high extinction coefficient, quantum yield and serum stability. Based on these observations, we investigated the impact of the fluorescent dye properties of conjugates 1-5 on tumour targeting and biodistribution. We aimed to identify the superior fluorophores for further development of fluorescent conjugates for FIGS, and our results suggest that ZW800-1 Forte is a potential candidate for conjugation to small molecule targeting ligands.

The fluorophores in this study differ with respect to net charge, hydrophilicity and spectral properties. ZW800-1 and ZW800-1 Forte, a more stable analogue of the former with a $\mathrm{C}-\mathrm{C}$ bond instead of the labile ether linkage on the meso carbon, are zwitterions that exhibit a balanced net charge of zero. This characteristic is expected to reduce non-specific binding to, e.g., serum proteins, and thus reduce the background signal [3, 32]. Indeed, zwitterionic NIR fluorophore-based targeted tracers have been shown to have favourable in vivo imaging characteristics, such as low background signal and rapid renal clearance in several preclinical studies [33], and have been clinically evaluated in patients with colon carcinoma [34]. In our study, conjugate 2 (ZW800-1 Forte) exhibited an intense fluorescence signal in tumours shortly after intravenous injection that was sustained for up to $48 \mathrm{~h}$ and it was the only conjugate that demonstrated significant differences between Skov-3 and A549. In contrast, there is a high non-specific fluorescent accumulation of 3 (IRDye $® 800 \mathrm{CW}$ ) at early time points in both cell line models, which obstructs clear distinction of the tumour signal, indicating that background reduction is pivotal for enhancing tumour contrast. IRDye ${ }^{\circledR} 800 \mathrm{CW}$ contains four sulfonate groups, giving it a high net negative charge. This negative charge results in a highly hydrophilic dye that interferes with the target binding properties of the conjugates, resulting in increased non-specific accumulation of the conjugate [3]. A study comparing IRDye ${ }^{\circledR} 800 \mathrm{CW}$ and ZW800-1, using cRGD as the target in preclinical melanoma models, shows similar tumour signal intensities, but a significant increase in TBR for the zwitterionic conjugate [26]. ICG-OSu and Cy7 possess one and no sulfonate group, respectively, and are thus more hydrophobic, which affects the clearance and circulation times of the folate conjugate [3]. Rapid renal clearance rates were observed in vivo for conjugates $\mathbf{1}, \mathbf{2}$ and $\mathbf{3}$, leading to a decrease in the fluorescence signal of FR $\alpha$ negative tissues [17, 33, 35]. ICGOSu-based conjugate $\mathbf{4}$ showed no accumulation in tumours and high biodistribution in the liver. This is also observed for the highly hydrophobic-free dye ICG, which is known to bind to plasma proteins, eliminating contrast agents larger 
than $8 \mathrm{~nm}$ via the hepatobiliary route [36, 37]. In addition, ICG exhibits limited photostability, which could explain the overall low fluorescence signal intensities obtained with ICG$\mathrm{OSu}$ [38]. In contrast, conjugate 5 began to accumulate in the subcutaneous tumour $4 \mathrm{~h}$ after administration, reaching maximum fluorescence signal intensity at $24 \mathrm{~h}$. We hypothesise that this could be the result of an unspecific EPR effect, following binding of $\mathbf{5}$ to albumin due to the presence of the meso-chlorine on the cyclohexenyl moiety [39]. The undesired low fluorescence intensity of $\mathbf{5}$ further limits its applicability for FIGS development. Lastly, conjugation of the different fluorophores to EDAF resulted in distinct absorption shifts with moderate bathochromic shifts for conjugates 1-4 and a hypsochromic shift for conjugate $\mathbf{5}$ in culture medium, compared to the literature values for the free dyes.

Optimal tumour contrast is critical for FIGS and can be achieved with both low background fluorescence, through reduction of non-specific binding, and high tumour-specific fluorescent signal, through improved targeting. Fluorescent conjugates targeting FR $\alpha$ are employed in late-stage clinical studies and have demonstrated favourable contrast for ovarian cancer FIGS, leading to improved surgical outcome [8, $40,41]$. However, in our study, the overall low TBR values obtained in subcutaneous ovarian cancer models could be due to the intermediate number of folate receptor antigen binding sites in the chosen cell line, Skov-3, or a potentially low binding affinity of the small molecule.

Distinct dyes exhibit excitation and emission spectra that require different in vivo imaging settings. While the preclinical IVIS Spectrum allows the user to adjust the excitation and emission filters, most intraoperative imaging systems do not have this function. Therefore, it is important to consider both the properties of the FIGS imaging system and the contrast agent. In our study, the properties of the intraoperative camera $\left(\lambda_{\text {ex }}=760 \pm 3 \mathrm{~nm}, \lambda_{\text {em }}=>781 \mathrm{~nm}\right)$ are most favourable for conjugate $2\left(\lambda_{\mathrm{ex}}=760 \mathrm{~nm}, \lambda_{\mathrm{em}}=788 \mathrm{~nm}\right)$ compared to the other conjugates, which have higher excitation wavelengths. Thus, the right combination of fluorophore, targeting ligand, linker and imaging system is necessary for increased tumour sensitivity and specificity, to ultimately achieve superior optical contrast.

\section{Conclusions}

Our results confirm that the physicochemical properties of fluorophores have a major impact on the biodistribution of the conjugates and the subsequent tumour contrast. The IRDye ${ }^{\circledR} 800 \mathrm{CW}$ conjugate 3 showed a high in vivo background fluorescence and was not sensitive enough to assess the differences in FR $\alpha$ expression between Skov-3 and A549. Although IRDye ${ }^{\circledR} 800 \mathrm{CW}$ is the most commonly employed agent for clinical use, we have demonstrated that other fluorescent contrast agents should be taken into consideration. ZW800-1 Forte has demonstrated potential as fluorescent contrast agent for targeted strategies providing specific fluorescence signal.

Supplementary Information The online version contains supplementary material available at https://doi.org/10.1007/s11307-021-01685-y.

Acknowledgements The authors thank Bjarte Holmelid for the technical support with the HRMS spectra acquisition. The authors would also like to thank Mireia Mayoral Safont and Constance de Villardi de Montlaur for technical assistance with the animal work. The authors thank Arild Holth, Department of Pathology, Oslo University Hospital, for performing the immunostaining. The authors would like to thank Mark Bordo from Curadel LCC for technical support and help with the intraoperative imaging system. Flow cytometry analysis was performed at the Flow Cytometry Core Facility, Department of Clinical Science, University of Bergen. Optical imaging experiments were carried out at the Molecular Imaging Center (MIC) and all the in vivo work was performed at the animal facility in Vivarium, Department of Clinical Medicine, University of Bergen.

Author Contribution E.G.J. and K.K. contributed equally to this work. E.G.J., K.K and E.M.C designed the study and performed the in vitro and in vivo work. V.F. participated in the in vivo work. B.D. scored the immunostains. E.G.J. and K.K. wrote the manuscript; V.F., E.M.C., L.B. and B.E.H. contributed to writing and editing the manuscript.

Funding Open access funding provided by University of Bergen (incl Haukeland University Hospital) This study was funded by the University of Bergen (815900), the H2020 program MSCA-ITN (675743), Helse Vest RHF and Helse Bergen HF $(911809,911852,912171,240222)$ and The Norwegian Cancer Society (182735) and The Research Council of Norway through its Centers of excellence funding scheme (223250, 262652). This work was partly supported by the Research Council of Norway through the Norwegian NMR Platform, NNP (226244).

\section{Declarations}

Conflict of Interest The authors declare that they have no conflict of interest in relation to the submitted work. Outside the submitted work, Dr. Bjørge reports grants from AstraZeneca, personal fees from AstraZeneca, other from AstraZeneca and GSK.

Open Access This article is licensed under a Creative Commons Attribution 4.0 International License, which permits use, sharing, adaptation, distribution and reproduction in any medium or format, as long as you give appropriate credit to the original author(s) and the source, provide a link to the Creative Commons licence, and indicate if changes were made. The images or other third party material in this article are included in the article's Creative Commons licence, unless indicated otherwise in a credit line to the material. If material is not included in the article's Creative Commons licence and your intended use is not permitted by statutory regulation or exceeds the permitted use, you will need to obtain permission directly from the copyright holder. To view a copy of this licence, visit http://creativeco mmons.org/licenses/by/4.0/.

\section{References}

1. McCormack E, Silden E, West RM et al (2013) Nitroreductase, a nearinfrared reporter platform for in vivo time-domain optical imaging of metastatic cancer. Cancer Res 73:1276-1286

2. Fonnes T, Strand E, Fasmer KE et al (2020) Near-infrared fluorescent imaging for monitoring of treatment response in endometrial carcinoma patient-derived xenograft models. Cancers (Basel) 12:370

3. Choi HS, Nasr K, Alyabyev S et al (2011) Synthesis and in vivo fate of zwitterionic near-infrared fluorophores. Angew Chem Int Ed Eng 50:6258-6263

4. ter Weele EJ, Terwisscha van Scheltinga AGT, Linssen MD et al (2016) Development, preclinical safety, formulation, and stability of clinical 
grade bevacizumab-800CW, a new near infrared fluorescent imaging agent for first in human use. Eur J Pharm Biopharm 104:226-234

5. Low PS, Singhal S, Srinivasarao M (2018) Fluorescence-guided surgery of cancer: applications, tools and perspectives. Curr Opin Chem Biol 45:64-72

6. du Bois A, Reuss A, Pujade-Lauraine E et al (2009) Role of surgical outcome as prognostic factor in advanced epithelial ovarian cancer: a combined exploratory analysis of 3 prospectively randomized phase 3 multicenter trials: by the Arbeitsgemeinschaft Gynaekologische Onkologie Studiengruppe Ovarialkarzinom (AGO-OVAR) and the Groupe d'Investigateurs Nationaux Pour les Etudes des Cancers de l'Ovaire (GINECO). Cancer 115:1234-1244

7. Tipirneni KE, Warram JM, Moore LS et al (2017) Oncologic procedures amenable to fluorescence-guided surgery. Ann Surg 266:36-47

8. van Dam GM, Themelis G, Crane LMA et al (2011) Intraoperative tumor-specific fluorescence imaging in ovarian cancer by folate receptor-alpha targeting: first in-human results. Nat Med 17:1315-1319

9. Hoogstins CES, Tummers QRJG, Gaarenstroom KN et al (2016) A novel tumor-specific agent for intraoperative near-infrared fluorescence imaging: a translational study in healthy volunteers and patients with ovarian cancer. Clin Cancer Res 22:2929

10. Kalli KR, Oberg AL, Keeney GL, Keeney GL, Christianson TJH et al (2008) Folate receptor alpha as a tumor target in epithelial ovarian cancer. Gynecol Oncol 108:619-626

11. Markert S, Lassmann S, Gabriel B, Klar M et al (2008) Alpha-folate receptor expression in epithelial ovarian carcinoma and non-neoplastic ovarian tissue. Anticancer Res 28:3567-3572

12. Hekman MCH, Boerman OC, Bos DL et al (2017) Improved intraoperative detection of ovarian cancer by folate receptor alpha targeted dual-modality imaging. Mol Pharm 14:3457-3463

13. Vergote I, Armstrong D, Scambia G et al (2016) A randomized, doubleblind, placebo-controlled, phase III study to assess efficacy and safety of weekly farletuzumab in combination with carboplatin and taxane in patients with ovarian cancer in first platinum-sensitive relapse. J Clin Oncol 34:2271-2278

14. Scaranti M, Cojocaru EA-O, Banerjee S, Banerji UA-O (2020) Exploiting the folate receptor $\alpha$ in oncology. Nat Rev Clin Oncol 17:349-359

15. FindLaw (1994-2001) FindLaw: Legal Professionals page. Accessed 22 May 2001.

16. Debie P, Hernot S (2019) Emerging fluorescent molecular tracers to guide intra-operative surgical decision-making. Front Pharmacol 10:510

17. Hernot S, van Manen L, Debie P et al (2019) Latest developments in molecular tracers for fluorescence image-guided cancer surgery. Lancet Oncol 20:e354-e367

18. Poellinger A, Burock S, Grosenick D et al (2011) Breast cancer: earlyand late-fluorescence near-infrared imaging with indocyanine green-a preliminary study. Radiology 258:409-416

19. Riaz A, Shreedhar B, Kamboj M, Natarajan S (2013) Methylene blue as an early diagnostic marker for oral precancer and cancer. SpringerPlus 2:95

20. Keereweer S, Mol IM, Kerrebijn JDF et al (2012) Targeting integrins and enhanced permeability and retention (EPR) effect for optical imaging of oral cancer. J Surg Oncol 105:714-718

21. Jiang JX, Keating JJ, Jesus EM et al (2015) Optimization of the enhanced permeability and retention effect for near-infrared imaging of solid tumors with indocyanine green. Am J Nucl Med Mol Imaging 5:390-400

22. Sano K, Nakajima T, Ali T et al (2013) Activatable fluorescent cysdiabody conjugated with indocyanine green derivative: consideration of fluorescent catabolite kinetics on molecular imaging. J Biomed Opt 18:101304

23. Watanabe R, Sato K, Hanaoka H et al (2014) Minibody-indocyanine green based activatable optical imaging probes: the role of short polyethylene glycol linkers. ACS Med Chem Lett 5:411-415
24. Ito S, Muguruma N, Hayashi S et al (1998) Development of agents for reinforcement of fluorescence on near-infrared ray excitation for immunohistological staining. Bioorg Med Chem 6:613-618

25. Debie P, Van Quathem J, Hansen I et al (2017) Effect of dye and conjugation chemistry on the biodistribution profile of near-infraredlabeled nanobodies as tracers for image-guided surgery. Mol Pharm $14: 1145-1153$

26. Choi HS, Gibbs SL, Lee JH et al (2013) Targeted zwitterionic nearinfrared fluorophores for improved optical imaging. Nat Biotechnol $31: 148-153$

27. Wang X, Huang SS, Heston WDW et al (2014) Development of targeted near-infrared imaging agents for prostate cancer. Mol Cancer Ther 13:2595

28. Corbett CJ, Frenzel Sulyok LG, Predina JD et al (2020) Comparison of a short versus long stokes shift near-infrared dye during intraoperative molecular imaging. Mol Imaging Biol 22:144-155

29. Schindelin J, Arganda-Carreras I, Frise E et al (2012) Fiji: an opensource platform for biological-image analysis. Nat Methods 9:676-682

30. Kleinmanns K, Fosse V, Davidson B et al (2020) CD24-targeted intraoperative fluorescence image-guided surgery leads to improved cytoreduction of ovarian cancer in a preclinical orthotopic surgical model. EBioMedicine 56:102783

31. Buckle T, van Willigen DM, Spa SJ et al (2018) Tracers for fluorescence-guided surgery: how elongation of the polymethine chain in cyanine dyes alters the pharmacokinetics of a dual-modality c[RGDyK] tracer. J Nucl Med 59:986

32. Sato K, Gorka AP, Nagaya T et al (2016) Role of fluorophore charge on the in vivo optical imaging properties of near-infrared cyanine dye/ monoclonal antibody conjugates. Bioconjug Chem 27:404-413

33. Handgraaf HJM, Boonstra MC, Prevoo HAJM et al (2017) Real-time near-infrared fluorescence imaging using cRGDZW800-1 for intraoperative visualization of multiple cancer types. Oncotarget 8:21054-21066

34. de Valk KS, Deken MM, Handgraaf HJM et al (2020) First-in-human assessment of cRGD-ZW800-1, a zwitterionic, integrin-targeted, near-infrared fluorescent peptide in colon carcinoma. Clin Cancer Res 26:3990-3998

35. Joshi BP, Wang TD (2018) Targeted optical imaging agents in cancer: focus on clinical applications. Contrast Media Mol Imaging 2018:2015237

36. Cherrick GR, Stein SW, Leevy CM, Davidson CS (1960) Indocyanine green: observations on its physical properties, plasma decay, and hepatic extraction. J Clin Invest 39:592-600

37. Lee JH, Park G, Hong GH et al (2012) Design considerations for targeted optical contrast agents. Quant Imaging Med Surg 2:266-273

38. Luo S, Zhang E, Su Y et al (2011) A review of NIR dyes in cancer targeting and imaging. Biomaterials 32:7127-7138

39. Usama SM, Park GK, Nomura S et al (2020) Role of albumin in accumulation and persistence of tumor-seeking cyanine dyes. Bioconjug Chem 31:248-259

40. Tummers QR, Hoogstins CE, Gaarenstroom KN et al (2016) Intraoperative imaging of folate receptor alpha positive ovarian and breast cancer using the tumor specific agent EC17. Oncotarget 7:32144-32155

41. Randall LM, Wenham RM, Low PS et al (2019) A phase II, multicenter, open-label trial of OTL38 injection for the intra-operative imaging of folate receptor-alpha positive ovarian cancer. Gynecol Oncol 155:63-68

Publisher's note Springer Nature remains neutral with regard to jurisdictional claims in published maps and institutional affiliations. 